\title{
IXe CONGRÈS INTERNATIONAL POUR LA QUARANTAINE ET LA PROTECTION DES PLANTES
}

(Moscou, 12-22 août 1958)

Périodiquement, les Délégués des services compétents des gouvernements de l'Est européen et de plusieurs pays d'Asie se réunissent pour rendre compte de leurs activités dans le domaine de la protection des Plantes et de la Quarantaine. Cette année, au congrès de Moscou, les trois organisations internationales, F.A.O., O.E.P.P. et C.I.L.B. étaient représentées.

Il n'y a pas lieu de commenter ici les importantes résolutions prises par les délégués gouvernementaux en vue d'intensifier et de coordonner les progrès techniques dans les domaines de la protection des Plantes et de la Quarantaine. Mais nous avons été particulièrement heureux de constater le désir des délégués d'élargir les bases de la coopération internationale, principalement avec l'O.E.P.P. et la C.I.L.B. : dans les résolutions du Congrès, il est envisagé de créer des groupes de travail similaires aux nôtres pour les pays de l'Est et avec lesquels les représentants qualifiés de la C.I.L.B. pourraient établir une liaison technique fructueuse.

Le point 20 des résolutions du Congrès de Moscou est ainsi exprimé : The conference seems it desirable to expand ties of countries participating in the present Conference with International Organizations in the field of Plant Protection and mainly reith E.P.P.O. and C.I.L.B.

Des visites techniques très documentées ont permis aux congressistes, hôtes du gouvernement de l'U.R.S.S., d'apprécier l'activité des divers laboratoires d'U.R.S.S. :

- Laboratoires spécialisés de l'Institut national de recherches phytosanitaires (V.I.Z.R.) de Leningrad et de leurs annexes, souvent situées près des stations de Quarantaine et qui se consacrent aux recherches théoriques;

- Laboratoires spécialisés à Moscou et en province dépendant du Laboratoire central de Quarantaine des Plantes agricoles (C.K.L.) de Moscou et chargés de résoudre les problèmes pratiques de Lutte biologique;

- Laboratoires spécialisés des Républiques comme ceux d'Ukraine à Kiev et d'Ouzbekistan à Tashkent parmi les plus importants.

Enfin, nous sommes très reconnaissants au gouvernement de l'U.R.S.S. pour sa très large hospitalité et l'excellente organisation d'un Congrès où des contacts techniques profitables ont été établis.

P. Grison. 